\title{
UNIVERSA MEDICINA
}

\section{Imaging of common bile duct stones}

\begin{abstract}
A Nurman
ABSTRACT

The gallbladder serves as the repository for bile produced in the liver. However, bile within the gallbladder may become supersaturated with cholesterol, leading to crystal precipitation and subsequent gallstone formation. Gallstone is one of the most common gastrointestinal diseases in clinical practice. Common bile duct stone may be silent and symptomless; alternatively the stone can cause acute cholangitis with jaundice, pain and fever and acute pancreatitis. Imaging of the gallbladder is typically requested for evaluation of right upper quadrant pain in patients with or without fever and jaundice. Hence,imaging is central to the investigation and diagnoses of choledocholithiasis. There are many options in the field of imaging of choledocholithiasis from a simple to more sophisticated examinations. Ultrasonography (US) has been the traditional modality for evaluating gallbladder disease, primarily owing to its high sensitivity and specificity for both stone disease and gallbladder inflammation. However, US is limited by patient body habitus, with degradation of image quality and anatomic detail in obese individuals. With the advent of faster and more efficient imaging techniques, magnetic resonance (MR) imaging has assumed an increasing role as an adjunct modality for gallbladder imaging. MR imaging allows simultaneous anatomic and physiologic assessment of the gallbladder and biliary tract. Magnetic resonance cholangiopancreatography is excellent for identifying the presence and the level of biliary obstruction. With newer diagnostic imaging technologies emerging, endoscopic retrograde cholangiopancreatography is evolving into a predominantly therapeutic procedure.
\end{abstract}

Keywords : Bile duct stone, ultrasound, cholangiography, magnetic resonance imaging
*Department of Internal Medicine, Medical Faculty, Trisakti University

\section{Correspondence}

${ }^{a} d r$. Rizaldy Pinzon

Department of Internal

Medicine, Medical Faculty, Trisakti University

Jl. Kyai Tapa 260 Grogol

Jakarta 11440

Tel 021-5672731 ext.2707

E-mail: nurman

_achmad@yahoo.com

Univ Med 2009; 28: 59-68

\section{INTRODUCTION}

Gallstones is one of the most common gastro intestinal diseases in clinical practice. Gallstones are found in about $10 \%$ of the general population, are twice as common in women as in men, and become more prevalent with increasing age. Most patients with gallstones are asymptomatic and discovered after routine general check up or for evaluation 
of patients with upper abdominal pain or with disturbance of liver function test. Laboratory test are helpful but results are not specific for the diagnosis of choledocholithiasis. ${ }^{(1,2)}$ Ultrasonography (US) is the technique of choice to detect gall bladder stone having a sensitivity of approximately $99 \%$ and a specificity of $99 \% .^{(3)}$ They are seen as echogenic foci within the gall bladder and cast accoustic shadows.

Choledocholithiasis is the present of stone in the common bile duct (CBD); the CBD stones are missed frequently by US (sensitivity 15$40 \%$ ). Unlike the gallbladder stone, CBD stone only produce accoustic shadow in about $20 \%$ of cases. ${ }^{(3)}$ The detection of CBD stone is impeded by the presence of gas in the duodenum, possible reflection and retraction of the sound beam of the duct and the location of the duct beyond the optimal foci of the transducer. On the other hand, CBD dilatation is identified accurately with up to $90 \%$ accuracy. ${ }^{(4)}$ It is therefore another options are needed to detect and confirm CBD stone.

\section{Clinical syndrome}

Choledocholithiasis may be silent and symptomless, discovered only by imaging at the time of a routine cholecystectomy for chronic calculous cholecystitis. Alternatively, the stone may cause an acute cholangitis with jaundice, pain and fever. ${ }^{(5)}$ A history of cholelithiasis is not essential for the diagnosis of choledocholithiasis because gall bladder stone can be asymptomatic. Pain is the most presenting symptom. The pain is colicky in nature, moderate in severity and located in in right upper quadrant of the abdomen. Jaundice occur when the CBD becomes obstructred and conjugated bilirubin enters the blood stream. A history of clay colored stools and tea colored urine, the jaundice can be episodic. ${ }^{(6)}$ Specific findings upon physical examination are few and are principally abdominal tenderness found the right upper quadrant of the abdomen and jaundice.

Laboratory test are helpful, but the results are not specific for the diagnoses of of choledocholithiasis because some patients are often asymptomatic and laboratory test results can be completely normal. The laboratory examination may revealed disturbance of the liver function test ie the elevation of serum bilirubin, gamma-glutamyl transpeptidase/ alkaline phosphatase and transaminase.

Patients with cholangitis and pancreatitis have abnormal laboratory values: i) white cell blood count elevation indicate the presence of infection or inflammation; ii) serum bilirubin level elevation indicates obstruction of the CBD; iii) alkaline phosphatase and gammaglutamyl transpeptidase levels are elevated. These tests results have a good predictive value for the presence of CBD stone and iv) liver transaminase (serum glutamic-pyruvic transaminase and oxaloacetic transaminase) levels are elevated.

Multiple modalities permit depiction of the normal anatomy as well as disease processes of the cystic duct, including i) plain photo of the abdomen; ii) abdominal US; iii) endoscopic ultrasound; iv) abdominal computed tomography scan (CT scan); v) endoscopic retrograde cholangio pancreography (ERCP); vi) percutaneous transhepatic cholangiography (PTC); vii) intra operative cholangiography (IOC); and viii) magnetic resonance cholangio pancreatogarphy (MRCP).

\section{Plain photo of the abdomen}

Abdominal plain photos often play an important role in the evaluations of patients with abdominal pain. ${ }^{(7)}$ For detecting CBD stones, however, the diagnostic yield is low and this test is tending to be omitted. It may reveal gall bladder stones, a calcified gall bladder, 
pancreatic calcification or rarely outline of a distended gall bladder. ${ }^{(6)}$ The wall of the gall bladder may also calcified (porcelain gall bladder). Gas in the biliary tree (aerobilia) may be seen after endoscopic sphincterotomy or surgical bile duct of bowel anastomosis. Bile duct dilatation and bile duct stones however, could not be seen by plain photo of the abdomen.

\section{Ultrasonography}

This is a non invasive, inexpensive, and readily modality for assessment of the bilary tree. It is usually the first modality used in the diagnoses of patients with biliary related symptoms. ${ }^{(6)}$ USG findings are accurate in the diagnoses of gallbladder stones (97\%) in elective situations (Figure 1) and $80 \%$ in the presence of acute cholecystitis), but CBD stones are missed frequently (sensitivity 15-40\%).

Right upper quadrant pain and/or jaundice and disturbance of liver function test are the most common indications for imaging of the biliary tract. US is the recommended initial imaging test in the evaluation of these patients. Based on

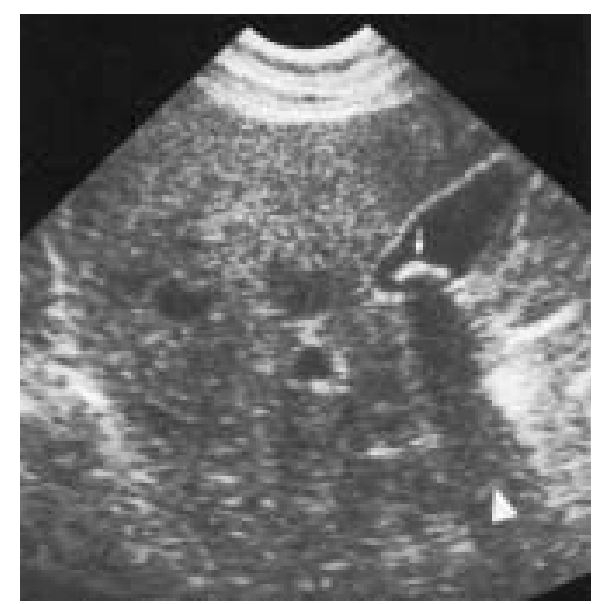

Figure 1. Gall bladder stone (arrow) with associated accoustic shodows (arrow head) clinical circumsrances, the differential diagnosis includes choledocholithiasis, biliary stricture or tumor. This is a non invasive, inexpensive, and readily modality for assessment of the bilary tree. US is very sensitive in the detection of biliary conctriction and stone diseases, allhough less sensitive for the detection of obstructing tumors, including pancreatic carcinoma and cholangiocarcinoma.

It is usually the first modality used in the diagnoses of patients with biliary related symptoms US is the most commonly used modality in the evaluation of gallstone disease, with a high specificity (95\%) and sensitivity (95\%) for stones larger than $2 \mathrm{~mm} .{ }^{(8,9)}$ The accuracy of sonography in detecting choledocholithiasis approximates $70 \%$ although detection of intrahepatic biliary ductal calculi by sonography is more problematic.

The biliary ductal system and gallbladder is best imaged with the patient fasted for eight to twelve hours. The fasting state achieves 2 objects, first, an empty stomach and duodenum to enble better imaging of the distal CBD and a relatively distended gall bladder to enable adequate study of the gall bladder wall and inraluminal contents.

Recognition of dilated bile ducts is central to sonographic imaging of the biliary system. Dilated intrahepatic bile ducts are greater than $2 \mathrm{~mm}$ in diameter and run parallel to the portal veins in the portal triads resulting in the appearance "parallel channel sign". The normal extra hepatic bile duct is 7 to $8 \mathrm{~mm}$ in diameter and usally wider in the subsegment than at the portal hepatic (Figure 2). Conditions that may mask biliary dilatation include pneumobilia of intrahapatic ducts when the posterior margin of a dilated duct is obscured by acoustic shadowing and the presence of biliary sludge or infected bile which may make the dilated ducts relatively invisible on sonography. 


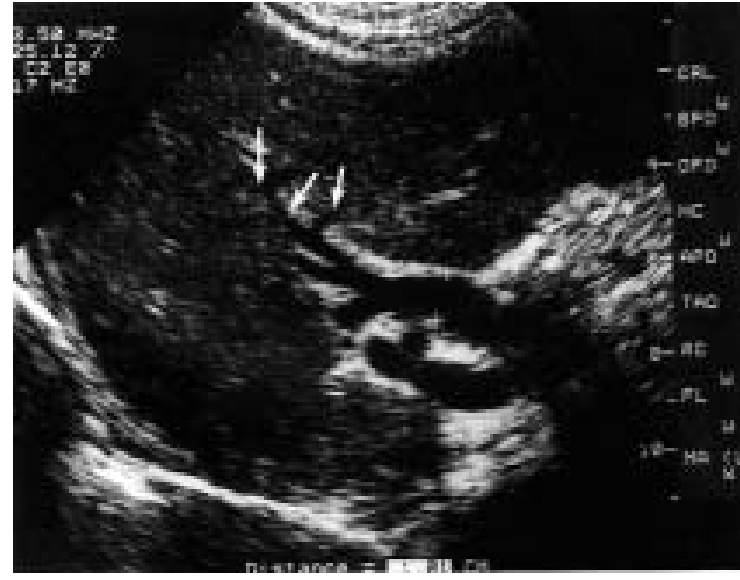

Figure 2. Ultra sound scan showing dilated intra-hepatic (arrowed) and common bile duct (marked ++ )

False-negative studies for biliary dilatation and/or small choledocholithiasis are usually because of overlying bowel gas obscuring the subhepatic common bile duct or to the absence of biliary dilatation in the presence of small non obstructing calculi.Common duct dilatation may not occur for 24 hours after initial stone impaction and intrahepatic duct may not dilate for up 72 hours after impaction.

Post cholecystectomy patients may have a relatively distended CBD of greater than 8 $\mathrm{mm}$ in diameter without mechanical obstruction. ${ }^{(10)}$ In such patients presenting with acute right upper quadrant pain who have obstructive liver chemistry or develop jaundice, a negative sonographic study should be followed by a cholangiographic examination, either magnetic resonance cholangio pancreatography (MRCP) or endoscopic retrograde pancreatography (ERCP, the latter supplemented by endoscopic ultrasound (EUS), to evaluate ductal calculi.

\section{Endosonography}

Transcutaneous ultrasonography is frequently hampered by interfering intestinal or pulmonal gas, bone or thick layers of fat tissue The deficiences in conventional imaging techniques prompted the development of endoscopy combined with ultrasonography (endoscopic ultrasound/EUS) in the early of 1980s in an effort to obtain more information about diseases of the gastrointestinal and its surroundings. ${ }^{(11)}$ Transcutaneous ultrasonography detects more than $95 \%$ of gallbladder stone in the bile duct by imaging from the descending bulb or duodenal bulb. ${ }^{(12)}$ CBD stones can be identified when US is used to evaluate the patients with a dilated bile duct or jaundice of unknown cause. If a common duct stone is suspected from transcutaneous imaging procedure or on clinical grounds, ERCP should be undertaken rather than EUS because it allows removal of the stone; however, EUS appears to be more accurate with less invasiveness. ${ }^{(13,14)}$ The ability to place an ultrasonographic probe in close proximity to suspected abnormalities, obviating the effects of gas, fat, and bone, enables EUS to yield a highresolution view of the gastrointestinal tract and adjacent structures. Several studies have compared the accuracy of EUS in detection of CBD stones with ERCP, and CT. ${ }^{(13,14)}$ In these reports, the sensitivity of EUS ranged from $88 \%$ to $97 \%$, with specificity of $96 \%$ to $100 \%$, which is comparable with ERCP and much better than CT. Moreover, the accuracy of EUS is better than ERCP for the detection of small stones. Small stones play an important role in acute pancreatitis and biliary-type pain. ${ }^{(15)}$

Conventional US is routinely used in the detection of cholelithiasis but it may not be able to detect small stones. There has been an interest in the role of EUS in detecting microlithiasis and in evaluating patients with idiopathic pancreatitis. Microlithiasis, occult common bile duct stones, small pancreatic neoplasm and pancreas divisum 
can be found in $75 \%$ of this population. ${ }^{(16,17)}$ Due to the lower risk of complication, EUS should be considered as an alternative to ERCP in this group of patients.

\section{Computed Tomogram (CT) Scan}

Using current CT technology and intravenous contrast enhanced (Figure 3), normal intra hepatic bile ducts may be visualized in over $40 \%$ of normal patients; these should not be confused with ductal dilatation. ${ }^{(18)}$ The extra hepatic bile ducts are identified normally in $80 \%$ of patients at CT and usually have diameters $4-10 \mathrm{~mm} .{ }^{(19)}$ The extra hepatic bile tree may be up to $10 \mathrm{~mm}$ in diameter in patients who have undergone cholecystectomy.

The presence or abscence of bile duct stones can be determined on the basis of the four criteria peviously described by Baron. ${ }^{(20)}$ With the target sign, the stone is seen as a central density surrounded by hypoattenuating bile or ampullary soft tissue. With the the rim sign one can see a faint rim of increased density along the margin of a low-density area. A calculous with increased density that is surrounded by a crescent of hypoattenuating bile is suggestive of the crescent sign. Indirect signs, such as the abrupt termination of the duct

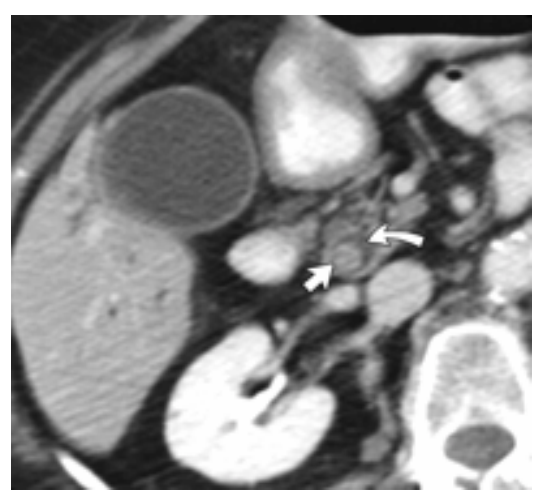

Figure 3. Common bile duct stone on contrast-enhanced CT scan.(straight arrow) or ductal dilatation, can be helpful. CT is comparable to US in its ability to detect biliary obstruction and exceeds US in the ability to predict the level and cause of biliary obstruction, although some controversies still exists. ${ }^{(21)}$ Most patients with obstructive jaundice will undergo US with CT used as a problem solving modality. The reported CT sensitivity for CBD stone detection varies from $45 \%$ to $90 \% .^{(22)}$ The ability to thin collimation and overlapping image reconstruction available with volume data acquisition of helical CT scanners improves stone detection rate. Non contrast CT may improve the recognition of choledocholithiasis to more than $90 \% .^{(23)}$ In dilated ducts it is easier to visualized stones. The CT appearance of CBD stones parallels that of gall stones and depend on the chemical composition for each stone. Densely calcified stones can be seen as high attenuation structures within the duct lumen, but most duct stones are is attenuating with soft tissue or bile on CT scan. Several processes can simulate duct stones on CT and care must be taken to avoid misdiagnoses. Pancreatic calculi, oral contrast in an adjacent duodenal diverticulum, residual contras material from prior cholangiogram, and papillary ductal neoplasm can simulate choledocholithiasis.

\section{Endoscopic Retrograde Cholangio Pancreatography (ERCP)}

Endoscopic retrograde cholangiopancreaticography is the standard therapy for the therapy of biliary obstruction. However, the success rate is not $100 \%$, depending on various patient and physician related factors. ${ }^{(24)}$ The CBD was canulated through the ampulla, contrast material is injected and films are obtained. Complications are hyperamilassemia and cholangitis. In most patients, ERCP is the modality of choice when choledocholithiasis is suggested. 


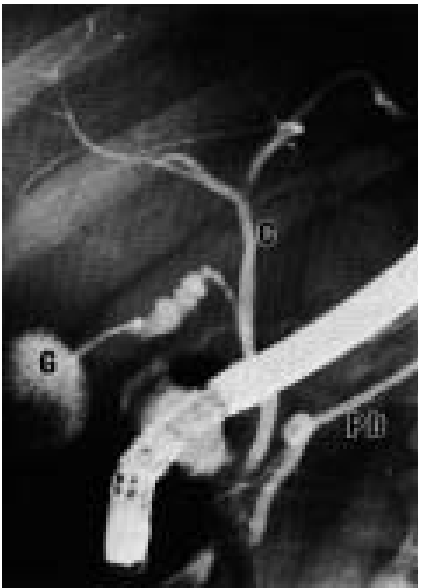

Figure 4. ERCP, normal appearance

$\mathrm{PD}=$ pancreatic duct, $\mathrm{G}=$ gall bladder, $\mathrm{C}=$ common bile duct

ERCP is a diagnostic procedure to diagnose problem in the liver, gall bladder, bile ducts and the pancreas in which X-ray is combined with the use flexible scope. With this procedure papilla of Vater is searched endoscopically, followed by canulation of the catheter and contrast is injected (Figure 4). This method needs a high skill ability. From ERCP, the blockade and stone in the bile duct, leakage of bile or pancreatic duct, pancreatic obstruction or stenosis, the existence of tumor may be found.

In most patients, ERCP is the modality of choice for diagnosing and treating choledocholithiasis when choledocholithiasis is suspected but carries potential risks of complications (Figure 5). Complications that occur were morbidity in $1-3 \%$, mortality in $0,2 \%$, and these were directly in connection with skills and experiences of the operator and to the underlying biliary and pancreatic diseases. The complications might occur are acute pancreatitis, cholangitis and bleeding particularly from sphincterotomy sites and duodenal perforation. ${ }^{(6,25)}$ Prophylactic antibiotics are often recommended, especially

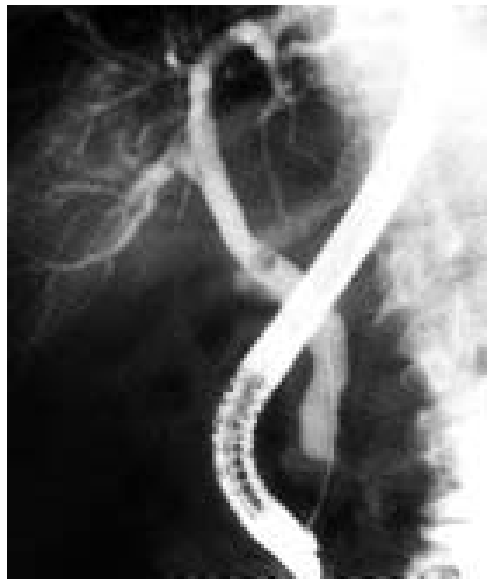

Figure 5. ERCP showing common bile duct stone. Asphincterotome hasbeen passed into the lower end of the bile duct

in patients with CBD obstruction. ERCP should be reserved for therapeutic purposes for those patients with a high likelihood of stones being present despite negative findings on prior studies.

\section{Percutaneous Transhepatic Cholangiography} (PTC)

In PTC intrahepatic biliary duct is directly punctured with fine flexible Chiba needle with the diameter $0.7 \mathrm{~mm}$ (22 Gauge), so that the patient still can normally breath during the procedure (Figure 6) ${ }^{(6)}$ US is used as a guidance in which the puncture was performed into the dilated bile duct. This method is easy and will $100 \%$ success if the bile duct is dilated. PTC may be the modality of choice in whom ERCP is difficult and failed due to the technical difficulties or when ERCP is not possible to perform i.e. in patients with hepaticoenterostomy, post Billroth II or in Klatskin tumor.

Complication occured less than $5 \%$ and consisted of bleeding, bile peritonitis and sepsis. ${ }^{(6)}$ Less common were pneumothorax and puncture of viscera. These complications can be reduced by ensuring fastidious technique, 


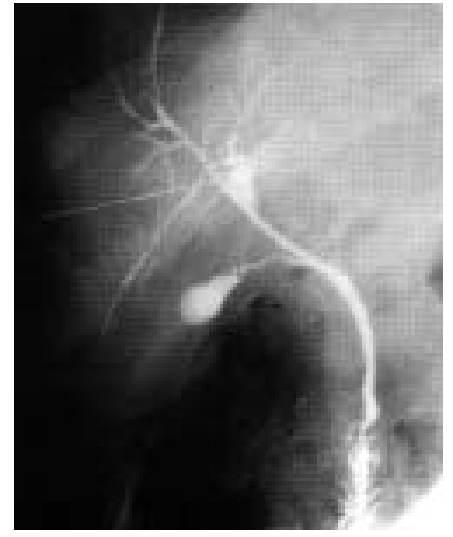

Figure 6. Diagnosis percutaneous transhepatic cholangiogram showing normal right and life intrahepatic ducts and common bile duct and free flow of contrast into duodenum. The gail bladder is beginning to fill

with a single puncture of the liver capsule, avoid over distention of ducts which may allow reflux of infected bile to the circulation through hepatic sinusoid, and ensuring that maneuver are carried out in suspended respiration. ${ }^{(26)}$

Uncorrected coagulopathy is a contraindication for PTC, and the normal size of the intrahepatic duct makes the procedure difficult. Prophylactic antibiotics are recommended to reduce the risk of cholangitis.

\section{Intraoperative Cholangiography (IOC)}

IOC is cholangiography that performs during surgical procedure. The most common indication of IOC is determination the need for exploration of CBD at the time of cholecystectomy. ${ }^{(6,26)}$ IOC is performed by inserting a catheter intra operatively into the cystic duct, followed by injecton of diluted $(50 \%)$ contrast material to outline biliary tree. ${ }^{(1)}$ Films are taken and are assessed for the precence of filling defects, the anatomy and the calibre tree, and the flow of contrast into the duodenum. This procedure can be performed at open or laparoscopic cholecystectomy (Figure 7). ${ }^{(27)}$

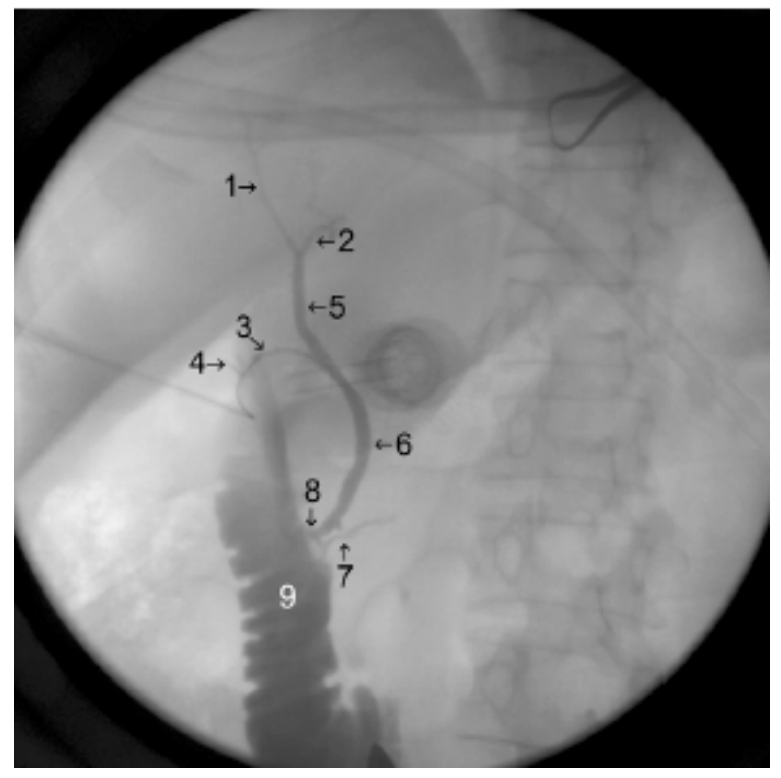

Figure 7. Normal intraoperative cholangiogram

(1) Right hepatic duct; (2) left hepatic duct; (3) cystic duct; (4) two surgical clips holding the cholangio catheter in the cystic duct; (5) common hepatic duct; (6) common bile duct; (7) pancreatic duct; (8) ampulla of Vater; (9) duodenum

IOC findings have a positive predictive value of $60-75 \%$ for the detection of CBD stones. The procedure can fail due to i) inability to cannulate the cystic duct, ii) the leakage of contrast during the injection, iii) air bubbles mimicking stones, iv) contrast flowing too quickly into the duodenum, preventing proper filling of the biliary tree, and v) spasm of the sphincter of Oddi. Postoperative cholangiography through a T-tube is indicated to ensure that all stones have been removed following exploration of the bile duct. ${ }^{(26)}$

In patients who have no history of pancreatitis, normal liver function test and CBD stone less than $10 \mathrm{~mm}$ diameter, laparoscopic cholecystectomy performed without intra operative chlolangiography does not result in an increased incidence of retained stone. ${ }^{(28)}$ 
Magnetic resonance cholangio pancreatography (MRCP)

MRCP was first described by Walner et al in $1991^{(29)}$ is a noninvasive method of imaging the biliary and pancreatic ducts. The basic principle underlying MRCP is that body fluids such as bile and pancreatic secretions, have high signal intensify in heavily $\mathrm{T} 2$-weighted magnetic resonance (ie they appear white), where as background tissues such as the surrounding liver and flowing blood generated little signal (ie they appear dark). As a result of this combination of imaging characteristics, MRCP provides optimal contrast between the hyperintense signal of the bile and pancreatic secretions and the hypointense signal of the background tissue (solid organs), and blood vessels have no measurable signal. On these images the intrinsic fluids of the biliary and pancreatic ducts give the cholangiogram and pancreatogram.

No special patient preparation is required but the usual contraindication to MR scanning apply. Patients with cardiac pacemaker, neurostimulator, ferromagnetic aneurism clips are excluded. The examination is usually performed after fasting for several hours. No sedation is required, no hospital admittance is needed. ${ }^{(30-32)}$ In some patients claustrophobia might be a problem. Oral contrast agent are not administered, no intravenous contrast agents are needed, and there is no radiation exposure. It is an ideal imaging method for patients with allergies to iodine based contrast or those with a general history of atopy, and prevent the occurrence of contrast nephropathy. ${ }^{(31)}$

MRCP is an alternative to diagnostic ERCP for the imaging of the bile tree and the pancreatic ducts (Figure 8). It is almost as good as ERCP in the diagnoses of CBD stones, though the ability of MRCP to detect small stones in a non dilated ducts may be limited. ${ }^{(33)}$ It is highly sensitive $(97,9 \%)$ and specific
$(84,4 \%)$ for choledocholithiasis and avoids the need for invasive imaging in most patients with suspected choledocholithiasis (Figure 9). ${ }^{(34)}$

MRCP can be considered as a new gold standard for the investigation of CBD stones and permits reservation of ERCP to patients with a high probability of therapeutic intervention. ${ }^{(35)}$ The European Association of Endoscopic Surgeons consider MRCP to be a standard test for patients with an intermediate probability of CBD stones. ${ }^{(36)}$ A major feature of MRCP is that is not a therapeutic procedure, while in contrast ERCP is used for both diagnoses and treatment. ${ }^{(30)}$

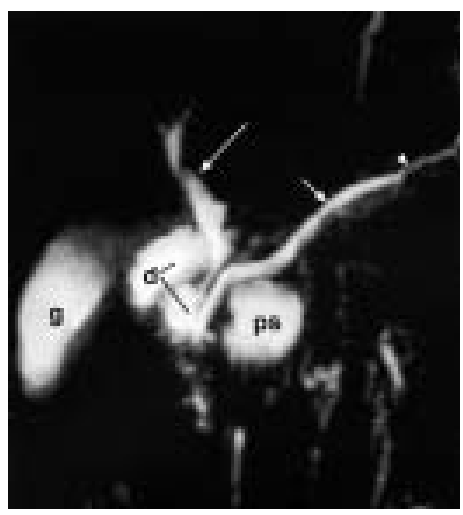

Figure 8. Normal MR cholangiography

Large arrow $=$ common bile duct, $\mathrm{g}=$ gall bladder, $\mathrm{d}=$ duodenum, small arrow $=$ pancreatic duct

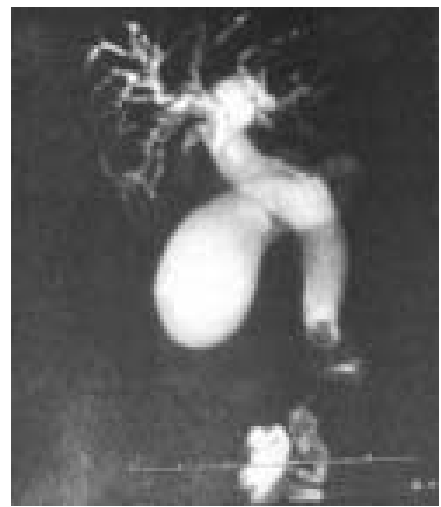

Figure 9. MRCP in patient with distal common bile duct stone 
In MRCP there is no morbidity nor mortality like in ERCP/PTC. More over MRCP is an important choice in patients where ERCP failed. ${ }^{(37)} \mathrm{MRCP}$ is a non invasive tool that is suitable in patients suspected to have pancreatico biliary diseases where there is no likelyhood or little possibility to perform therapeutic intervention e.g. patients with asymptomatic cholelithiasis without clinical evidence of clinical bile duct disease like the presence of jaundice and abnormalities of liver function test.

\section{CONCLUSION}

CBD stone may be silent and symptomless; alternatively the stone can cause right upper quadrant abdominal pain with jaundice, pain and fever and acute pancreatitis. Imaging is central to the investigation and diagnoses of choledocholithiasis. Sonography is recommended as initial imaging and from the result further imaging techniques can be chosen. CT may become more important and a competitor against MRCP in the near future because of improvements in the detectors and the software. For imaging of the biliary system, MRCP has replaced invasive ERC and percutaneous transhepatic cholangiography as the method of choice. With newer diagnostic imaging technologies emerging, ERCPis evolving into a predominantly therapeutic procedure.

\section{REFERENCES}

1. Dandan IS, Soweid AM, Abiad F. Choledocholithiasis: overview. eMedicine Gastroenterology; 2008. Available at: http:// emedicine.medscape.com/article/172216-overview. Accessed November 8, 2008.

2. Catalana OA, Sahani DV, Kalva SP, Cushing MS, Hahn PF, Brown JJ, et al. MR imaging of the gallbladder: a pictorial essay. Radio Graphics 2008; 28: 135-55.
3. Yeh BM, Breiman RS, Taouli B. Biliary tract depiction in living potential liver donors: comparison of conventional MR, mangafodipir trisodium-enhanced excretory MR, and multidetector row CT cholangiography-initial experience. Radiology 2004; 230: 645-1.

4. Turner MA, Fulcher AS. The cystic duct: normal anatomy and disease processes. Radio Graphics 2001; 21: 3-22.

5. Eisen GM, Dominitz JA, Faigel DO, Goldstein JL, Kalloo AN, Petersen BT, et al. An annotated algorithm for the evaluation of choledocholithiasis. Gastrointest Endosc 2001; 53: 864-66.

6. Sherlock S, Dooley J. Gallstones and inflammatory gallbladder diseases. In: Sherlock S, Dooley J, editors. Diseases of the liver and biliary system. $11^{\text {th }}$ ed. Malden, Mass: Blackwell; 2002. p. 597628.

7. Gupta K, Bhandari RK, Chander R. Comparative study of plain film abdomen and ultrasound in nontraumatic acute abdomen. Int J Radiol Imaging 2005; 15: 109-15.

8. Fowley WD, Quiroz FA. The role of sonography in imaging of the biliary tract. Ultrasound Q 2007; 23: 123-5.

9. Stroszcynski C, Hunerbein M. Malignant biliary obstruction: value of imaging findings. Abdom Imaging 2005; 30: 314-23.

10. Khalid TR, Casillas VJ, Montalvo BM, Centeno R, Levi JU. Using MR cholangiopancreatographyto evaluate iatrogenic bile duct injury. Am J Roentgenol 2001; 177: 1347-52.

11. Rosch T, Lorenz R, Suchy R. Colonic endoscopic ultrasonography: first results of a new technique. Gastrointest Endosc 1990; 36: 382-6.

12. Yoshitsugu K, Makoto T, Shin Y, Nobuyuki S, Massaki S, Shoichiroh T, et al. Diagnosis of common bile duct calculi with intraductal ultrasonography during endoscopic biliary cannulation. J Gastroenterol Hepatol 2002; 17: 70812.

13. Kohut M, Nowakowska-Dulawa E, Marek T, Kaczor R, Nowak A. Accuracy of linear endoscopic ultrasonography in the evaluation of patients with suspected common bile duct stones. Endoscopy 2002; 34: 299-303.

14. Buscarini E, Tansini P, Vallisa D, Zambelli A, Buscarini L. EUS for suspected choledocholithiasis: do benefits outweigh costs? A prospective, controlled study. Gastrointest Endosc 2003; 57: $510-8$. 
15. Venneman NG, Renooij W, Rehfeld JF, VanbergeHenegouwen GP. Small gallstones, preserved gallbladder motility, and fast crystallization are associated with pancreatitis. Hepatology 2005; 41: 738-6.

16. Tandon M, Topazian M. Endoscopic ultrasound in idiopathic acute pancreatitis. J Gastroenterol 2001; 96: 705-9.

17. Frossard JL, Soca-Valencia, Amouyal G, Marty O, Hadenque A, Aouyal J. Usefulness of endoscopic ultrasonography in patients with "idiopathic" acute pancreatitis. Am J Med 2000; 109: 196-200.

18. Liddell RM, Baron RL, Ekstrom JE. Normal intrahepatic bile duct: CT depiction. Radiology 1990; 176: 633-5.

19. Schulte SJ, Baron RL, Teefy SA. CT of the extrahepatic bile ducts: wall thickness and contrast enhancement in normal and abnormal ducts. Am J Roentonolog 1990; 154: 79-85.

20. Baron RL. Common bile duct stones: reassessment of criteria for CT diagnoses. Radiology 1987; 162: 419-24.

21. Upadhyaya V, Upadhyaya DN, Ansari MA, Shilka VK. Comparative assessment of imaging modalities in biliary obstruction. In J Radiol Imag 2006; 16: 577-82.

22. Knowlton JQ, TaylorAJ, Reichelderfer M, Stang J. Imaging of biliary tract inflammation: an update. Am J Roentonolog 2008; 190: 984-92.

23. Soto JA, Alvarez O, Múnera F, Velez SM, Valencia J, Ramírez N. Diagnosing bile duct stones: comparison of unenhanced helical CT, oral contrast-enhanced CT cholangiography, and MR cholangiography. Am J Roentgenol 2000; 175: 1127-34.

24. Puspok A. Biliary therapy: are we ready for EUSguidance? Minerva Med 2007; 98: 379-84.

25. Topal B, Van de Moortel M, Fieuws S, Vanbeckevoort $\mathrm{D}$. The value of magnetic resonance cholangio-pancreatography in predicting common bile duct stones in patients with gall stones diseases. Br J Surg 2003; 90: $42-7$.

26. Karani J. The biliary tract. In: Sutton D, editor. Texbook of radiology imaging vol.I. London: Churchill Livingstone; 2003. p. 711-36.
27. Massarweh NN, Flum DR. Role of intraoperative cholangiography in avoiding bile duct injury. J Am Coll Surg 2007; 10: 656-64.

28. McFarlane MEC, Thomas CAL, McCartney T. Selective operative cholangiography in the performance of laparoscopic cholecystectomy. Int J Clin Pract 2005; 59: 1301-3.

29. Wallner WK, Schumacher KA, Weidemaier W, Friedrich JM. Dilated biliary tract: evaluation with magnetic resonance cholangio pancreatography with a T2-weighted contrast-enhanced fast sequence. Radiology 1991; 18: 805-8.

30. McEneaney P, Mitchell MT, McDermoth R. Update on magnetic resonance cholangio pancreatography. Gastroenterol Clin N Am 2002; 31: 731-46.

31. Calco MM, Bufanda L, Calderon A, Heras I, Cabriada JL, Bernal A, et al. Role of magnetic resonance cholangio pancreatography in patients with suspected choledocholithiasis. Mayo Clin Proc 2002; 77: 422-8.

32. Romagnuolo J, Bardon M, Rahme E, Joseph L, Reinhold C, Barkun AN. Magnetic resonance cholangio pancreatography: a meta analysis of test performance in suspected biliary disease. Ann Inter Med 2003; 139-7: 547-57.

33. Williams EJ, Green J, Beckingham I, Park R, Martin $\mathrm{D}$, Lombard M. Guidelines of the management of common bile duct stones (CBDS). Gut 2008; 57: 1004-21.

34. Shanmugan V, Beattie GC, Jule SR, Reid W, Loudon MA. Is Magnetic Resonance Cholangio Pancreatography the new gold standard in biliary imaging ?. Br J Radiol 2005; 78: 888-93.

35. Gore RM, Yaghmai V, Newmark GM, Berlin JW, Miller FH. Imaging benign and malignant disease of the gallbladder. Radiol Clin North Am 2002; 40: 1307-23.

36. Neugebauer E, Sauerland S, Fingerhut A. European Association Endoscopic Surgery guidelines for endoscopic surgery. Berlin: Springer; 2006.

37. Leung J. Fundamentals in ERCP in advanced digestive endoscopy: endoscopic retrograde cholangio pancreatography. In: Cotton PB, Leung J, editors. $1^{\text {st }}$ ed. Massachusets: Blackwell Publishing; 2006. p. 17-80. 